MATHEMATICS OF COMPUTATION

Volume 75, Number 256, October 2006, Pages 2021-2035

S 0025-5718(06)01885-0

Article electronically published on July 11, 2006

\title{
ON THE MINIMAL POLYNOMIAL OF GAUSS PERIODS FOR PRIME POWERS
}

\author{
S. GURAK
}

AbStract. For a positive integer $m$, set $\zeta_{m}=\exp (2 \pi i / m)$ and let $\mathbf{Z}_{m}^{*}$ denote the group of reduced residues modulo $m$. Fix a congruence group $H$ of conductor $m$ and of order $f$. Choose integers $t_{1}, \ldots, t_{e}$ to represent the $e=\phi(m) / f$ cosets of $H$ in $\mathbf{Z}_{m}^{*}$. The Gauss periods

$$
\theta_{j}=\sum_{x \in H} \zeta_{m}^{t_{j} x} \quad(1 \leq j \leq e)
$$

corresponding to $H$ are conjugate and distinct over $\mathbf{Q}$ with minimal polynomial

$$
g(x)=x^{e}+c_{1} x^{e-1}+\cdots+c_{e-1} x+c_{e} .
$$

To determine the coefficients of the period polynomial $g(x)$ (or equivalently, its reciprocal polynomial $G(X)=X^{e} g\left(X^{-1}\right)$ ) is a classical problem dating back to Gauss. Previous work of the author, and Gupta and Zagier, primarily treated the case $m=p$, an odd prime, with $f>1$ fixed. In this setting, it is known for certain integral power series $A(X)$ and $B(X)$, that for any positive integer $N$

$$
G(X) \equiv A(X) \cdot B(X)^{\frac{p-1}{f}}\left(\bmod X^{N}\right)
$$

holds in $\mathbf{Z}[X]$ for all primes $p \equiv 1(\bmod f)$ except those in an effectively determinable finite set. Here we describe an analogous result for the case $m=p^{\alpha}$, a prime power $(\alpha>1)$. The methods extend for odd prime powers $p^{\alpha}$ to give a similar result for certain twisted Gauss periods of the form

$$
\psi_{j}=i^{*} \sqrt{p} \sum_{x \in H}\left(\frac{t_{j} x}{p}\right) \zeta_{p^{\alpha}}^{t_{j} x} \quad(1 \leq j \leq e)
$$

where $(\bar{p})$ denotes the usual Legendre symbol and $i^{*}=i^{\frac{(p-1)^{2}}{4}}$.

\section{INTRODUCTION}

For any positive integer $m$, set $\zeta_{m}=\exp (2 \pi i / m)$ and let $\mathbf{Z}_{m}^{*}$ denote the group of reduced residues modulo $m$. Fix a congruence group $H$ defined modulo $m$ of order $f$ and conductor $m$. Choose integers $t_{1}=1, t_{2}, \ldots, t_{e}$ to represent the $e=\phi(m) / f$ cosets of $H$ in $\mathbf{Z}_{m}^{*}$. The Gauss periods

$$
\theta_{j}=\sum_{x \in H} \zeta_{m}^{t_{j} x} \quad(1 \leq j \leq e)
$$

corresponding to $H$ lie in the subfield $K$ of $\mathbf{Q}\left(\zeta_{m}\right)$ fixed by the Galois actions induced by sending $\zeta_{m} \rightarrow \zeta_{m}^{x}$ for $x \in H$. They are conjugate and distinct over $\mathbf{Q}$

Received by the editor June 2, 2005.

2000 Mathematics Subject Classification. Primary 11L05, 11T22, 11T23.

(C)2006 American Mathematical Society 
8, 11, and have minimal polynomial

$$
g(x)=g_{H}(x)=x^{e}+c_{1} x^{e-1}+\cdots+c_{e-1} x+c_{e}
$$

of degree $e$. For any numerical character $\chi$ defined modulo $m$, the Gauss sum $G(\chi)$ is given by

$$
G(\chi)=\sum_{x \in \mathbf{Z}_{m}^{*}} \chi(x) \zeta_{m}^{x} .
$$

The Gauss periods (1) are intimately related with the sums (3); namely,

$$
G(\chi)=\sum_{j=1}^{e} \chi\left(t_{j}\right) \theta_{j}
$$

for any character $\chi$ annihilating $H$, and

$$
\theta_{j}=\frac{1}{e} \sum_{\chi} \bar{\chi}\left(t_{j}\right) G(\chi) \quad(1 \leq j \leq e),
$$

where $\chi$ runs through the characters defined modulo $m$ which annihilate $H$. (Here $\bar{\chi}$ denotes the multiplicative inverse of the $\chi$.)

It is well known from the theory of equations 4 that the coefficients $c_{r}$ of $g(x)$ in (2) can be computed in terms of the symmetric power sums $S_{n}=\sum\left(\theta_{j}\right)^{n}$ using Newton's identities

$$
\begin{gathered}
S_{r}+c_{1} S_{r-1}+\cdots+c_{r-1} S_{1}+r c_{r}=0 \quad(1 \leq r \leq e), \\
S_{n}+c_{1} S_{n-1}+\cdots+c_{e-1} S_{n-e+1}+c_{e} S_{n-e}=0 \quad(n>e) .
\end{gathered}
$$

Alternatively, if

$$
G(X)=G_{H}(X)=X^{e} g\left(X^{-1}\right)=1+c_{1} X+\cdots+c_{e} X^{e}
$$

is the reciprocal polynomial of $g(x)$, its logarithm is formally expressed as

$$
\log G(X)=\log \prod_{j=1}^{e}\left(1-\theta_{j} X\right)=-\sum_{n=1}^{\infty} \frac{S_{n} X^{n}}{n}
$$

in terms of the power sums $S_{n}$.

To determine the coefficients of the period polynomial $g(x)$ in (2) (or equivalently its reciprocal polynomial in (7)) is a classical problem dating back to Gauss [5]. When $f=1$ one has $\theta_{1}=\zeta_{m}$ in (1) whose minimal polynomial is the well-known cyclotomic polynomial

$$
\begin{gathered}
\psi_{m}(x)=\prod_{d \mid m}\left(x^{d}-1\right)^{\mu(m / d)}, \\
\text { with } \psi_{p^{\alpha}}(x)=x^{p^{\alpha-1}(p-1)}+\cdots+x^{p^{\alpha}}+1
\end{gathered}
$$

when $m=p^{\alpha}$, a prime power. Henceforth, we shall assume $f>1$ throughout this paper.

Previous work of the author [7] and Gupta and Zagier [6] primarily treated the case $m=p$, an odd prime, with $f>1$ fixed. In this case, the beginning coefficients of $g(x)$ exhibit a polynomial dependence on $p$ for all sufficiently large primes $p \equiv 1(\bmod f)$. More precisely, Gupta and Zagier [6] showed for certain integral 
power series $A(X)$ and $B(X)$ depending only on simple arithmetic properties of the $f$-roots of unity $\zeta_{f}^{v}(1 \leq v \leq f)$, that for any positive integer $N$

$$
G(X) \equiv A(X) \cdot B(X)^{(p-1) / f} \quad\left(\bmod X^{N}\right)
$$

holds in $\mathbf{Z}[X]$ for all primes $p \equiv 1(\bmod f)$ except for those in an effectively determinable finite set. Moreover, they give an elegant algorithm to compute the exceptional sets. For $f=2$, the congruence (10) holds for $N=p$, so $G(X)$ is uniquely determined. Indeed,

$$
G(X)=\sum_{r=0}^{e}(-1)^{[r / 2]}\left(\begin{array}{c}
{[e-r / 2]} \\
{[r / 2]}
\end{array}\right) X^{r}
$$

exactly in closed form, a formula known to Gauss [5]. (Here [ ] denotes the greatest integer function.) Some extensions of these results for certain congruence groups $H$ of square-free conductor $m=p_{1} \cdots p_{r}$ with a fixed number of prime factors of specified types have been suggested (see $[8$ and also [6]). However, the prime power case $m=p^{\alpha}, \alpha>1$, seems to have been overlooked. It is this situation we describe here. The case $p=2$ is treated first, separately in Section 2, where closed form formulas are known for $G_{H}(X)$ for any congruence group $H$ of conductor $2^{\alpha}$. In Section 3 we give an analog of (10) for Gauss periods corresponding to congruence groups $H$ of odd prime power conductor $p^{\alpha}, \alpha>1$, together with an adaptation of Gupta and Zagier's algorithm to determine exceptional prime powers. When $f=2$ this congruence is shown to hold modulo $X^{p^{\alpha-1}}$, not enough though to completely determine $G(X)$. However, we have recently found [9] closed form formulas in this case for $G(X)$ which generalize (11). In the final section, we extend the results for odd prime powers $p^{\alpha}$ to certain twisted Gauss periods of the form

$$
\psi_{j}=i * \sqrt{p} \sum_{x \in H}\left(\frac{t_{j} x}{p}\right) \zeta_{p^{\alpha}}^{t_{j} x} \quad(1 \leq j \leq e)
$$

where $(\bar{p})$ denotes the usual Legendre symbol and $i *=i^{(p-1)^{2} / 4}$. Such quadratic twists or integer multiples of them arise classically as values of Kloosterman sums [10, 13] for odd prime powers $p^{\alpha}, \alpha>1$.

\section{GAuss PERIOdS FOR $2^{\alpha}$}

Throughout this section $H$ is a congruence group of conductor $2^{\alpha} \quad(\alpha>1)$ and order $f>1$. It is known that there are only two possible choices for $H$. For the sake of completeness we include a brief rationale for this fact.

Proposition 1. A congruence group $H$ of conductor $2^{\alpha}, \alpha>1$, and order $f>1$ must be either $\left\{1,2^{\alpha}-1\right\}$ or $\left\{1,2^{\alpha-1}-1\right\}$ modulo $2^{\alpha}$.

Proof. Since $H$ has conductor $2^{\alpha}$ with $f>1$, it is known from class field theory that the field $K$ corresponding to $H$ is a proper subfield of $\mathbf{Q}\left(\zeta_{2^{\alpha}}\right)$ not contained in $\mathbf{Q}\left(\zeta_{2^{\alpha-1}}\right)$, and so $\alpha>2$. Since $2 \mid f, H$ contains an element of order two modulo $2^{\alpha}$ - either $2^{\alpha}-1,2^{\alpha-1}-1$ or $2^{\alpha-1}+1$. The last choice can be eliminated since $H$ has conductor $2^{\alpha}$. Thus $K$ is contained in one of the cyclic fields $\mathbf{Q}\left(\zeta_{2^{\alpha}}+\zeta_{2^{\alpha}}^{-1}\right)$ or $\mathbf{Q}\left(\zeta_{2^{\alpha}}-\zeta_{2^{\alpha}}^{-1}\right)$ which correspond to the congruence groups $\left\{1,2^{\alpha}-1\right\}$ or $\left\{1,2^{\alpha-1}-1\right\}$ modulo $2^{\alpha}$, respectively. But any proper subfield of $\mathbf{Q}\left(\zeta_{2^{\alpha}}+\zeta_{2^{\alpha}}^{-1}\right)$ or $\mathbf{Q}\left(\zeta_{2^{\alpha}}-\zeta_{2^{\alpha}}^{-1}\right)$ is a subfield of $\mathbf{Q}\left(\zeta_{2^{\alpha-1}}+\zeta_{2^{\alpha-1}}^{-1}\right)$ contained in $\mathbf{Q}\left(\zeta_{2^{\alpha-1}}\right)$. Thus either $K=\mathbf{Q}\left(\zeta_{2^{\alpha}}+\zeta_{2^{\alpha}}^{-1}\right)$ or $\mathbf{Q}\left(\zeta_{2^{\alpha}}-\zeta_{2^{\alpha}}^{-1}\right)$, so $H$ equals $\left\{1,2^{\alpha}-1\right\}$ or $\left\{1,2^{\alpha-1}-1\right\}$. 
From the proposition above the only possibilities for $\theta_{1}$ in (1) here is $\zeta_{2^{\alpha}}+\zeta_{2^{\alpha}}^{-1}$ or $\zeta_{2^{\alpha}}-\zeta_{2^{\alpha}}^{-1}$, each with $f=2$ and $\alpha>2$. The corresponding minimal polynomials are classically known from the properties of Chebyshev polynomials. We quote results from [9] relevant to the discussion here.

Theorem 1. The minimal polynomial for $\zeta_{2^{\alpha}}+\zeta_{2^{\alpha}}^{-1}$ for $\alpha>2$ is equivalently characterized by

$$
g(x)=\sum_{n=0}^{2^{\alpha-3}}(-1)^{n} \frac{2^{\alpha-2}}{2^{\alpha-2}-n}\left(\begin{array}{c}
2^{\alpha-2}-n \\
n
\end{array}\right) x^{2^{\alpha-2}-2 n}
$$

or the power sums

$$
S_{n}= \begin{cases}2^{\alpha-2}\left(\begin{array}{c}
n \\
n / 2
\end{array}\right) & \text { if } n \text { is even, } \\
0 & \text { if } n \text { is odd, }\end{cases}
$$

for $1 \leq n \leq 2^{\alpha-2}$, or the congruence

$$
G(X) \equiv B_{+}(X)^{2^{\alpha-2}}\left(\bmod X^{2^{\alpha-1}}\right)
$$

where

$$
B_{+}(X)=\exp \left(-\sum_{n=1}^{\infty}\left(\begin{array}{c}
2 n \\
n
\end{array}\right) \frac{X^{2 n}}{2 n}\right)=\frac{1}{2}\left(1+\sqrt{1-4 X^{2}}\right) .
$$

The minimal polynomial for $\zeta_{2^{\alpha}}-\zeta_{2^{\alpha}}^{-1}$ for $\alpha>2$ is characterized equivalently by

$$
g(x)=\sum_{n=0}^{2^{\alpha-3}} \frac{2^{\alpha-2}}{2^{\alpha-2}-n}\left(\begin{array}{c}
2^{\alpha-2}-n \\
n
\end{array}\right) x^{2^{\alpha-2}-2 n}
$$

or the power sums

$$
S_{n}= \begin{cases}-2^{\alpha-2}\left(\begin{array}{c}
n \\
n / 2
\end{array}\right) & \text { if } 2 \| n \\
2^{\alpha-2}\left(\begin{array}{c}
n \\
n / 2
\end{array}\right) & \text { if } 4 \mid n \\
0 & \text { if } n \text { is odd, }\end{cases}
$$

for $1 \leq n \leq 2^{\alpha-2}$, or the congruence

$$
G(X) \equiv B_{-}(X)^{2^{\alpha-2}}\left(\bmod X^{2^{\alpha-1}}\right)
$$

where

$$
B_{-}(X)=\exp \left(-\sum_{n=1}^{\infty}(-1)^{n}\left(\begin{array}{c}
2 n \\
n
\end{array}\right) \frac{X^{2 n}}{2 n}\right)=\frac{1}{2}\left(1+\sqrt{1+4 X^{2}}\right) .
$$

We treat the case when $H$ has an odd prime power conductor next. 


\section{GAUSS PERIODS FOR ODD PRIME POWERS}

Throughout this section we assume the congruence group $H$ in (1) has conductor $m=p^{\alpha}$ with $p$ odd and $\alpha>1$. Since $\mathbf{Z}_{p^{\alpha}}^{*}$ is cyclic, $H=\left(\mathbf{Z}_{p^{\alpha}}^{*}\right)^{\phi\left(p^{\alpha}\right) / f}$, or equivalently $H$ equals the group of $f$-roots of unity in $\mathbf{Z}_{p^{\alpha}}^{*}$. In particular, since $H$ has conductor $p^{\alpha}, p \nmid f$ so $p \equiv 1(\bmod f)$. To compute the symmetric power sums $S_{n}$, we introduce certain counting functions $T_{n}\left(p^{\gamma}\right)$ for $0<\gamma \leq \alpha$ as in [8]. Specifically, let $T_{n}\left(p^{\gamma}\right)$ count the number of times

$$
x_{1}+\cdots+x_{n} \equiv 0\left(\bmod p^{\gamma}\right)
$$

for choice of tuples $\left(x_{1}, \ldots, x_{n}\right)$ with $x_{i}$ in $\left(\mathbf{Z}_{p^{\gamma}}^{*}\right)^{\phi\left(p^{\gamma}\right) / f} \quad(1 \leq i \leq n)$. These counting functions possess the following useful property.

Lemma 1. For $\gamma>1, T_{n}\left(p^{\gamma-1}\right)-T_{n}\left(p^{\gamma}\right)$ equals the number of tuples $\left(x_{1}, \ldots, x_{n}\right)$ with $x_{i}$ in $\left(\mathbf{Z}_{p^{\gamma}}^{*}\right)^{\phi\left(p^{\gamma}\right) / f}$ for which $p^{\gamma-1} \|\left(x_{1}+\cdots+x_{n}\right)$.

Proof. First observe that any $f$-root of unity $x \bmod p^{\gamma-1}$ lifts to a unique $f$-root of unity $x^{\prime} \bmod p^{\gamma}$, since

$$
\left(x+t p^{\gamma-1}\right)^{f} \equiv x^{f}+f t p^{\gamma-1} \equiv 1\left(\bmod p^{\gamma}\right)
$$

has a unique solution $t$ satisfying $-f t \equiv\left(x^{f}-1\right) / p^{\gamma-1}(\bmod p)$. Thus each solution $x_{1}+\cdots+x_{n} \equiv 0\left(\bmod p^{\gamma-1}\right)$ with $x_{i}$ in $\left(\mathbf{Z}_{p^{\gamma-1}}^{*}\right)^{\phi\left(p^{\gamma-1}\right) / f}$ lifts to a unique solution $x_{1}^{\prime}+\cdots+x_{n}^{\prime} \equiv 0\left(\bmod p^{\gamma-1}\right)$ with $x_{i}^{\prime}$ in $\left(\mathbf{Z}_{p^{\gamma}}^{*}\right)^{\phi\left(p^{\gamma}\right) / f}$. The statement of the lemma now readily follows.

Now observe in the expansion (8)

$$
\log G(X)=-\sum_{n=1}^{\infty} S_{n} X^{n} / n=-\sum_{n=1}^{\infty} \sum_{j=1}^{e}\left(\sum_{x \in H} \zeta_{p^{\alpha}}^{t_{j} x}\right)^{n} \frac{X^{n}}{n},
$$

that $S_{n}$ equals the inner sum

$$
\sum_{j=1}^{e}\left(\sum_{x \in H} \zeta_{p^{\alpha}}^{t_{j} x}\right)^{n}=\frac{1}{f} \sum_{r \in Z_{p^{\alpha}}^{*}} \sum_{x_{i} \in H} \zeta_{p^{\alpha}}^{r\left(x_{1}+\cdots+x_{n}\right)} .
$$

But in view of the lemma above, and since

$$
\begin{gathered}
\sum_{r \in Z_{p^{\alpha}}^{*}} \zeta_{p^{\alpha}}^{r x}= \begin{cases}\phi\left(p^{\alpha}\right) & \text { if } p^{\alpha} \mid x \\
-p^{\alpha-1} & \text { if } p^{\alpha-1} \| x \\
0 & \text { otherwise, }\end{cases} \\
\sum_{r \in Z_{p^{\alpha}}^{*}} \sum_{x_{i} \in H} \zeta_{p^{\alpha}}^{r\left(x_{1}+\cdots+x_{n}\right)}=\phi\left(p^{\alpha}\right) T_{n}\left(p^{\alpha}\right)-p^{\alpha-1}\left(T_{n}\left(p^{\alpha-1}\right)-T_{n}\left(p^{\alpha}\right)\right) \\
=p^{\alpha} T_{n}\left(p^{\alpha}\right)-p^{\alpha-1} T_{n}\left(p^{\alpha-1}\right) .
\end{gathered}
$$

Thus,

$$
S_{n}=\frac{p^{\alpha-1}}{f}\left(p T_{n}\left(p^{\alpha}\right)-T_{n}\left(p^{\alpha-1}\right)\right) .
$$

Now let $\beta_{f}(n)$ count the number of times $\epsilon_{1}+\cdots+\epsilon_{n}=0$ for $f$-roots of unity $\epsilon_{i}$ in $\mathbf{Q}\left(\zeta_{f}\right)$, so $T_{n}\left(p^{\alpha-1}\right) \geq T_{n}\left(p^{\alpha}\right) \geq \beta_{f}(n)$. Thus as long as $T_{n}\left(p^{\alpha-1}\right)=\beta_{f}(n)$,

$$
S_{n}=\frac{\phi\left(p^{\alpha}\right)}{f} \beta_{f}(n)
$$


above. Letting $\xi_{f}(n)$ be the set of odd prime powers $p^{\alpha}(\alpha>1), p \equiv 1(\bmod f)$, for which $T_{n}\left(p^{\alpha-1}\right)>\beta_{f}(n)$ and putting

$$
B(X)=\exp \left(-\sum_{n=1}^{\infty} \beta_{f}(n) \frac{X^{n}}{n}\right),
$$

one obtains from (8) and (15) the following analog of Gupta and Zagier's result 6. Theorem 1].

Theorem 2. For each natural number $N$, the congruence

$$
G(X) \equiv B(X)^{\phi\left(p^{\alpha}\right) / f} \quad\left(\bmod X^{N}\right)
$$

holds in $\mathbf{Z}[X]$ for all odd prime powers $p^{\alpha}(\alpha>1), p \equiv 1(\bmod f)$, except those lying in $\xi_{f}(n)$ for some $n<N$.

Before giving some examples, some comments concerning the result above and the computation of the exceptional sets $\xi_{f}(n)$ are in order. The power series $B(X)$ in (16) is the same integral power series appearing in [6. The counting function $\beta_{f}(n)$ has a nice expression when $f$ is a prime power or twice a prime (chiefly, Theorem 2 in [6]) In particular, one has

$$
\beta_{l}(n)= \begin{cases}0 & \text { if } l \not \backslash n \\ \frac{n !}{((n / l) !)^{l}} & \text { if } l \mid n\end{cases}
$$

when $l$ is a prime and

$$
\beta_{4}(n)= \begin{cases}0 & \text { if } n \text { is odd } \\
\left(\begin{array}{c}
n \\
n / 2
\end{array}\right)^{2} & \text { if } n \text { is even }\end{cases}
$$

(see also Corollary 2 in [8]).

Gupta and Zagier [6] give an elegant alternative expression for $\beta_{f}(n)$ which is useful in computing the exceptional sets $\xi_{f}(n)$. For any tuple $\bar{i}=\left(i_{1}, \ldots, i_{n}\right)$ in $(\mathbf{Z} / f \mathbf{Z})^{n}$, let $P_{\bar{i}}(x)$ denote the polynomial $x^{i_{1}}+\cdots+x^{i_{n}}$ with $i_{v}(1 \leq v \leq n)$ chosen to be the least nonnegative representative of the class $i_{v}(\bmod f)$. Then

$$
\beta_{f}(n)=\operatorname{card}\left(\left\{\bar{i}=\left(i_{1}, \ldots, i_{n}\right) \in(\mathbf{Z} / f \mathbf{Z})^{n} \mid \psi_{f}(x) \text { divides } P_{\bar{i}}(x)\right\}\right),
$$

where $\psi_{f}(x)$ denotes the $f$ th cyclotomic polynomial (9). If one fixes a prime $\tilde{p}$ lying above $p$ in $\mathbf{Q}\left(\zeta_{f}\right)$, then

$$
T_{n}\left(p^{\gamma}\right)=\operatorname{card}\left(\left\{\bar{i}=\left(i_{1}, \ldots, i_{n}\right) \in(\mathbf{Z} / f \mathbf{Z})^{n} \mid \tilde{p}^{\gamma} \text { divides } \zeta_{f}^{i_{1}}+\cdots+\zeta_{f}^{i_{n}}\right\}\right)
$$

in (13), independent of the choice of $\tilde{p}$. Clearly $T_{n}\left(p^{\alpha-1}\right)>\beta_{f}(n)$ if and only if for some tuple $\bar{i}=\left(i_{1}, \ldots, i_{n}\right)$ in $(\mathbf{Z} / f \mathbf{Z})^{n}$

$$
\tilde{p}^{\alpha-1} \mid\left(\zeta_{f}^{i_{1}}+\cdots+\zeta_{f}^{i_{n}}\right) \text { but } \zeta_{f}^{i_{1}}+\cdots+\zeta_{f}^{i_{n}} \neq 0 .
$$

Thus $\xi_{f}(n)$ consists of all odd prime powers $p^{\alpha}, p \equiv 1(\bmod f)$ for which $(21)$ holds for some tuple $\bar{i}$ in $(\mathbf{Z} / f \mathbf{Z})^{n}$. Now a necessary condition for $\bar{i}$ to satisfy (21) is $R_{\bar{i}} \equiv 0\left(\bmod p^{\alpha-1}\right)$, but $R_{\bar{i}} \neq 0$, where $R_{\bar{i}}$ denotes the resultant of $P_{\bar{i}}(x)$ and $\psi_{f}(x)$. The resultant $R_{\bar{i}}=0$ if $\psi_{f}(x)$ divides $P_{\bar{i}}(x)$. When $\psi_{f}(x) \not P_{\bar{i}}(x)$ (so $\left.\left(\psi_{f}, P_{\bar{i}}\right)=1\right), R_{\bar{i}}$ is essentially the smallest positive integer $R$ for which the equation $g(x) \psi_{f}(x)+h(x) P_{i}(x)=R$ is solvable with polynomials $g, h \in \mathbf{Z}[x]$, or equivalently, the norm $N_{\mathbf{Q}\left(\zeta_{f}\right) / \mathbf{Q}} P_{i}\left(\zeta_{f}\right)$. Thus to determine which prime powers lie in $\xi_{f}(n)$, one 
TABLE 1.

\begin{tabular}{|c|c|c|}
\hline \multicolumn{3}{|c|}{$f=4, n=7: \quad \tilde{p}_{5}=2-\zeta_{5}$ where $\zeta_{4} \equiv 7\left(\bmod \tilde{p}_{5}^{2}\right)$} \\
\hline$\overline{\bar{i}}$ & & $\zeta_{f}^{i_{1}}+\cdots+\zeta_{f}^{i_{n}} \quad$ factors \\
\hline 0000002 & 25 & $5=\left(2+\zeta_{4}\right)\left(2-\zeta_{4}\right)$ \\
\hline 0000111 & 25 & $4+3 \zeta_{4}=\zeta_{4}\left(2-\zeta_{4}\right)^{2}$ \\
\hline 0000333 & 25 & $4-3 \zeta_{4}=-\zeta_{4}\left(2+\zeta_{4}\right)^{2}$ \\
\hline 0111112 & 25 & $5 \zeta_{4}=\zeta_{4}\left(2+\zeta_{4}\right)\left(2-\zeta_{4}\right)$ \\
\hline \multicolumn{3}{|c|}{$f=5, n=7: \quad \tilde{p}_{11}=2+\zeta_{5}^{2}$ where $\zeta_{5} \equiv 3\left(\bmod \tilde{p}_{11}^{2}\right)$} \\
\hline $\bar{i}$ & $R_{\bar{i}}$ & $\zeta_{f}^{i_{1}}+\cdots+\zeta_{f}^{i_{n}} \quad$ factors \\
\hline 0000123 & 121 & $3-\zeta_{5}^{4}=-\zeta_{5}^{4}\left(1+\zeta_{5}^{4}\right)\left(2+\zeta_{5}^{3}\right)^{2}$ \\
\hline 0000124 & 121 & $3-\zeta_{5}^{3}=-\zeta_{5}^{3}\left(1+\zeta_{5}^{3}\right)\left(2+\zeta_{5}\right)^{2}$ \\
\hline 0000134 & 121 & $3-\zeta_{5}^{2}=-\zeta_{5}^{2}\left(1+\zeta_{5}^{2}\right)\left(2+\zeta_{5}^{4}\right)^{2}$ \\
\hline 0000234 & 121 & $3-\zeta_{5}=-\zeta_{5}\left(1+\zeta_{5}\right)\left(2+\zeta_{5}^{2}\right)^{2}$ \\
\hline 0001113 & 121 & $3+3 \zeta_{5}+\zeta_{5}^{3}=-\zeta_{5}^{2}\left(1+\zeta_{5}^{2}\right)\left(2+\zeta_{5}\right)\left(2+\zeta_{5}^{4}\right)$ \\
\hline 0001222 & 121 & $3+3 \zeta_{5}^{2}+\zeta_{5}=-\zeta_{5}^{4}\left(1+\zeta_{5}^{4}\right)\left(2+\zeta_{5}^{2}\right)\left(2+\zeta_{5}^{3}\right)$ \\
\hline \multicolumn{3}{|c|}{$f=3, n=11: \quad \tilde{p}_{7}=\zeta_{3}-2$ where $\zeta_{3} \equiv-19\left(\bmod \tilde{p}_{7}^{2}\right)$} \\
\hline$i$ & $R_{\bar{i}}$ & $\zeta_{f}^{i_{1}}+\cdots+\zeta_{f}^{i_{n}}$ factors \\
\hline 00000000111 & 49 & $8+3 \zeta_{3}=-\zeta_{3}^{2}\left(\zeta_{3}-2\right)^{2}$ \\
\hline 00000000222 & 49 & $8+3 \zeta_{3}^{2}=-\zeta_{3}\left(\zeta_{3}^{2}-2\right)^{2}$ \\
\hline
\end{tabular}

first restricts to those prime powers $p^{\alpha}, p \equiv 1(\bmod f)$ for which $R_{\bar{i}} \equiv 0\left(\bmod p^{\alpha-1}\right)$ but $R_{\bar{i}} \neq 0$ for some tuple $\bar{i}$ in $(\mathbf{Z} / f \mathbf{Z})^{n}$. For such a prime power $p^{\alpha}$, select a primitive $f$-root of unity $g$ modulo $p^{\alpha-1}$ for which $\tilde{p}^{\alpha-1} \mid\left(\zeta_{f}-g\right)$. Then in view of (21), $p^{\alpha} \in \xi_{f}(n)$ if and only if

$$
g^{i_{1}}+\cdots+g^{i_{n}} \equiv 0\left(\bmod p^{\alpha-1}\right) \text { with } \zeta_{f}^{i_{1}}+\cdots+\zeta_{f}^{i_{n}} \neq 0
$$

for some tuple $\bar{i}=\left(i_{1}, \ldots, i_{n}\right)$ in $(\mathbf{Z} / f \mathbf{Z})^{n}$. It is enough to consider tuples $\bar{i}$, inequivalent under translation and permutation.

Table 1 shows the results of this computation for finding exceptional prime powers $p^{3}$ among the inequivalent $n$-tuples $\bar{i}$ for which $R_{\bar{i}} \equiv 0\left(\bmod p^{2}\right), R_{\bar{i}} \neq 0$ in cases $(f, n)=(4,7),(5,7)$ and $(3,11)$.

To illustrate when $f=5$ and $n=7$, one finds six inequivalent classes of 7 -tuples $\bar{i}$ with $p^{2} \mid R_{\bar{i}}$ and $R_{\bar{i}} \neq 0$. Representatives for each such class are given in Table 1 , all have resultant $R_{\bar{i}}=121$. To check if $11^{3} \in \xi_{5}(7)$ one may choose $\tilde{p}_{11}=2+\zeta_{5}^{2}$, where $\zeta_{5} \equiv 3\left(\bmod \tilde{p}_{11}^{2}\right)$. One of these tuples satisfies $(21)$ with $\tilde{p}_{11}=2+\zeta_{5}^{2}$ and $\alpha=3$; namely, 0000234 , so $11^{3} \in \xi_{5}(7)$. There are $\frac{7 !}{4 !}=210$ permutations of 0000234 with five translations each, so

$$
T_{7}(121)=\beta_{5}(7)+210 \cdot 5=1250
$$

as $\beta_{5}(7)=0$.

The "new" exceptional prime powers for given $n$ are the elements of $\xi_{f}(n)$ which do not appear in $\xi_{f}\left(n^{\prime}\right)$ for some $n^{\prime}<n$. Table 2 lists the "new" exceptional prime powers for $3 \leq f \leq 8$ and small values of $n$. 
TABLE 2 .

\begin{tabular}{|r|l|l|l|}
\hline$\underline{n}$ & $f=3$ & $f=4$ & $f=6$ \\
\hline 3 & --- & $5^{2}$ & $7^{2}$ \\
4 & $7^{2}$ & --- & $13^{2}$ \\
5 & $13^{2}$ & $13^{2}, 17^{2}$ & $19^{2}$ \\
6 & --- & --- & $31^{2}$ \\
7 & $19^{2}, 31^{2}$ & $5^{3}, 29^{2}, 37^{2}$ & $37^{2}, 43^{2}$ \\
8 & $43^{2}$ & --- & $7^{3}$ \\
9 & --- & $41^{2}, 53^{2}$ & $61^{2}, 67^{2}, 73^{2}$ \\
10 & $37^{2}, 73^{2}$ & --- & $79^{2}$ \\
11 & $7^{3}, 67^{2}$ & $61^{2}, 73^{2}, 101^{2}$ & $97^{2}, 103^{2}$ \\
\hline \hline$\underline{n}$ & $f=5$ & $f=7$ & $f=8$ \\
\hline 3 & $11^{2}$ & $43^{2}$ & $17^{2}$ \\
4 & $61^{2}$ & $29^{2}, 71^{2}, 547^{2}$ & $41^{2}$ \\
5 & $41^{2}$ & $113^{2}, 197^{2}$, & $73^{2}, 89^{2}, 97^{2}$ \\
& & $421^{2}, 463^{2}$ & $113^{2}, 257^{2}$ \\
6 & $31^{2}, 71^{2}$ & $211^{2}, 379^{2}, 449^{2}$ & $137^{2}, 313^{2}$ \\
& $181^{2}, 521^{2}$ & $751^{2}, 2689^{2}$ & \\
7 & $11^{3}, 101^{2}, 151^{2}$ & $239^{2}, 281^{2}, 337^{2}$ & $17^{3}, 193^{2}, 233^{2}$ \\
& $191^{2}, 461^{2}$ & $1583^{2}, 1597^{2}$ & $241^{2}, 281^{2}, 337^{2}$ \\
& & & $353^{2}, 409^{2}, 433^{2}$, \\
& & & $641^{2}, 1297^{2}$ \\
\hline
\end{tabular}

Here are a couple of examples to illustrate Theorem 2.

Example 1. Consider the case $p=7$ with $f=3$, so

$$
\beta_{3}(n)= \begin{cases}n ! /((n / 3) !)^{3} & \text { if } 3 \mid n, \\ 0 & \text { otherwise }\end{cases}
$$

from (17) with

$$
B(X)=1-2 X^{3}-13 X^{6}-158 X^{9}-2431 X^{12}-\cdots
$$

in (16), The power 49 first appears in the exceptional set $\xi_{3}(4)$ with $T_{4}(7)=12>$ $\beta_{3}(4)=0$, whereas 343 first appears in the exceptional set $\xi_{3}(11)$ with $T_{11}(49)=$ $495>\beta_{3}(11)=0$. The minimal polynomials $G(X)$ for $\theta_{49}^{-1}=\left(\zeta_{49}+\zeta_{49}^{18}+\zeta_{49}^{-19}\right)^{-1}$ begins

$$
1-28 X^{3}+\underline{7} X^{4}+\underline{14} X^{5}+\underline{189} X^{6}+\cdots,
$$

whereas that for $\theta_{1}^{-1}=\left(\zeta_{343}+\zeta_{343}^{18}+\zeta_{343}^{-19}\right)^{-1}$ begins

$$
1-196 X^{3}+7^{2} \cdot 362 X^{6}-7^{3} \cdot 2872 X^{9}+\underline{7}^{2} \cdot \underline{15} X^{11}+7^{3} \cdot 109733 X^{12}+\cdots .
$$

The underscored coefficients deviate as expected from the pattern of the beginning coefficients given by Theorem 2 .

Example 2. Consider next $p=5$ with $f=4$, so $\beta_{4}(n)$ is given by (18) with

$$
B(X)=1-2 X^{2}-7 X^{4}-50 X^{6}-456 X^{8}-\cdots
$$

in (16). The power 25 first appears in $\xi_{4}(3)$ with $T_{3}(5)=12>\beta_{4}(3)=0$, whereas 125 first appears in the exceptional set $\xi_{4}(7)$ with $T_{7}(25)=140>\beta_{4}(7)=0$. Here 
one may take $H=\{ \pm 1, \pm 7\}$ for $m=25$ and $H=\{ \pm 1, \pm 57\}$ for $m=125$. The minimal polynomial $G(X)$ for $\theta_{1}^{-1}=\left(\zeta_{25}+\zeta_{25}^{-1}+\zeta_{25}^{57}+\zeta_{25}^{-57}\right)^{-1}$ is

$$
1-10 X^{2}+\underline{5} X^{3}+\underline{10} X^{4}+\underline{1} X^{5} .
$$

That for $\theta_{1}^{-1}=\left(\zeta_{125}+\zeta_{125}^{-1}+\zeta_{125}^{57}+\zeta_{125}^{-57}\right)^{-1}$ begins

$$
1-50 X^{2}+1025 X^{4}-11250 X^{6}+\underline{125} X^{7}+\cdots .
$$

The underscored coefficients deviate as expected from the pattern of the beginning coefficents given by Theorem 2 .

We conclude this section with a discussion of the case $f=2$ where a closed formula for $G(X)$ has been obtained [9]. The power series $B(X)$ in (16) is just

$$
B(X)=\frac{1}{2}\left(1+\sqrt{1-4 X^{2}}\right)=1-\sum_{n=0}^{\infty}\left(\begin{array}{c}
2 n \\
n
\end{array}\right) \frac{X^{2 n+2}}{n+1}
$$

with $\beta_{2}(n)$ given by (17). The minimal polynomial $G(X)$ for $\left(2 \cos 2 \pi / p^{\alpha}\right)^{-1}$ is seen to have the form

$$
G(X)=\left(\frac{1+\sqrt{1-4 X^{2}}}{2}\right)^{\phi\left(p^{\alpha}\right) / 2} \cdot \frac{1-\left(\frac{1-\sqrt{1-4 X^{2}}}{2 X}\right)^{p^{\alpha}}}{1-\left(\frac{1-\sqrt{1-4 X^{2}}}{2 X}\right)^{p^{\alpha-1}}}
$$

with power sums $S_{n}$ satisfying

$$
S_{n}=p^{\alpha} \sum_{t=1, t \text { odd }}^{\left[n p^{-\alpha}\right]}\left(\begin{array}{c}
n \\
\left(n-p^{\alpha} t\right) / 2
\end{array}\right)-p^{\alpha-1} \sum_{t=1, t \text { odd }}^{\left[n p^{1-\alpha}\right]}\left(\begin{array}{c}
n \\
\left(n-p^{\alpha-1} t\right) / 2
\end{array}\right)
$$

if $n$ is odd, or

$$
\frac{\phi\left(p^{\alpha}\right)}{2}\left(\begin{array}{c}
n \\
n / 2
\end{array}\right)+p^{\alpha} \sum_{t=1}^{\left[n p^{-\alpha} / 2\right]}\left(\begin{array}{c}
n \\
n / 2-p^{\alpha} t
\end{array}\right)-p^{\alpha-1} \sum_{t=1}^{\left[n p^{1-\alpha} / 2\right]}\left(\begin{array}{c}
n \\
n / 2-p^{\alpha-1} t
\end{array}\right)
$$

if $n$ is even. A closed form formula for $G(X)$ is

$$
G(X)=X^{\phi\left(p^{\alpha}\right) / 2}+\sum_{j=0}^{(p-3) / 2} X^{p^{\alpha-1} j} C_{p^{\alpha-1}\left(\frac{p-1}{2}-j\right)}(X),
$$

where

$$
C_{d}(X)=\sum_{n=0}^{[d / 2]}(-1)^{n} \frac{d}{d-n}\left(\begin{array}{c}
d-n \\
n
\end{array}\right) X^{2 n},
$$

or equivalently with coefficients in (7) satisfying for $1 \leq r<\phi\left(p^{\alpha}\right) / 2$,

$$
c_{r}=\sum_{j=0, j \equiv r}^{\left[r p^{1-\alpha}\right]}(-1)^{t_{j}} \frac{p^{\alpha-1}\left(\frac{p-1}{2}-j\right)}{p^{\alpha-1}\left(\frac{p-1}{2}-j\right)-t_{j}}\left(\begin{array}{c}
p^{\alpha-1}\left(\frac{p-1}{2}-j\right)-t_{j} \\
t_{j}
\end{array}\right)
$$

with $c_{\left.\phi\left(p^{\alpha}\right) / 2\right)}=\left(\frac{-2}{p}\right)$, where $t_{j}=\left(r-p^{\alpha-1} j\right) / 2$.

From (22) or (24), it follows that the congruence

$$
G(X) \equiv B(X)^{\phi\left(p^{\alpha}\right) / 2} \quad\left(\bmod X^{N}\right)
$$

holds in $\mathbf{Z}[X]$ for $N=p^{\alpha-1}$ but not for $N>p^{\alpha-1}$, and thus fails to determine $G(X)$ which is of degree $\phi\left(p^{\alpha}\right) / 2$. Indeed, one can show $p^{\alpha}$ first appears in the exceptional set $\xi_{2}(n)$ for $n=p^{\alpha-1}$. 


\section{Twisted Gauss Periods FOR $p^{\alpha}$}

Here we consider quadratic twists of Gauss periods for prime powers $p^{\alpha}, \alpha>1$. When $p=2$ with $\alpha>3$, the twisted Gauss periods have the form $\sqrt{2}\left(\zeta_{2^{\alpha}}^{v}+\zeta_{2_{\alpha}}^{-v}\right)$ or $\sqrt{2}\left(\zeta_{2^{\alpha}}^{v}-\zeta_{2^{\alpha}}^{-v}\right)$ for some odd integer $v$, and their corresponding minimal polynomials are easily obtained from the Theorem 1 in Section 2. With no loss of generality then we restrict attention to the case in which $p$ is odd, and consider the twisted Gauss periods

$$
\psi_{j}=i^{*} \sqrt{p} \sum_{x \in H}\left(\frac{t_{j} x}{p}\right) \zeta_{p^{\alpha}}^{t_{j} x} \quad(1 \leq j \leq e),
$$

with $H$ a congruence group of conductor $p^{\alpha}(\alpha>1)$ and order $f>1$ as in (1). The twisted Gauss periods (26) also lie in the subfield $K$ of $\mathbf{Q}\left(\zeta_{p^{\alpha}}\right)$ corresponding to $H$ with $[K: \mathbf{Q}]=\phi\left(p^{\alpha}\right) / f$. In fact $\psi_{j}=\operatorname{Tr}_{\mathbf{Q}\left(\zeta_{p^{\alpha}}\right) / K}\left(i^{*} \sqrt{p} \zeta_{p^{\alpha}}^{t_{j}}\right)(1 \leq j \leq e)$, and each is seen to generate $K$.

Proposition 2. Each twisted Gauss period $\psi_{j}$ in (26) generates the field $K$ over Q.

Proof. It suffices to show the conjugates $\psi_{j}(1 \leq j \leq e)$ are all distinct. For this purpose set

$$
T(\chi)=\sum_{j=1}^{e} \chi\left(t_{j}\right) \psi_{j}
$$

for any numerical character $\chi$ annihilating $H$. Then

$$
\psi_{j}=\frac{1}{e} \sum_{\chi} \bar{\chi}\left(t_{j}\right) T(\chi) \quad(1 \leq j \leq e),
$$

the sum taken over the characters $\chi$ annihilating $H$. Generalizing the argument in the Appendix of [8] one finds in view of the lemma there that the $\psi_{j} \quad(1 \leq j \leq e)$ will be distinct provided $T(\chi) \neq 0$ for all $\chi$ annihilating $H$ with conductor $f(\chi)$ satisfying $\left(p^{\alpha} / f(\chi), f(\chi)\right)=1$, where $p^{\alpha} / f(\chi)$ is square-free. We assert that this hypothesis above holds here so that the $\psi_{j}$ are distinct. Indeed for any character $\chi$ annihilating $H, T(\chi)$ equals

$$
\sum_{j=1}^{e} \chi\left(t_{j}\right) i^{*} \sqrt{p} \sum_{x \in H}\left(\frac{t_{j} x}{p}\right) \zeta_{p^{\alpha}}^{t_{j} x}=i^{*} \sqrt{p} \sum_{x \in Z_{p^{\alpha}}^{*}} \chi(x)\left(\frac{x}{p}\right) \zeta_{p^{\alpha}}^{x},
$$

or $i^{*} \sqrt{p} G(\chi \psi)$ in terms of a Gauss sum (3), where $\psi$ is the quadratic character $\left(\frac{x}{p}\right)$ considered modulo $p^{\alpha}$. But any such character $\chi$ with $\left(p^{\alpha} / f(\chi), f(\chi)\right)=1$ and $p^{\alpha} / f(\chi)$ square-free must have conductor $f(\chi)=p^{\alpha}$. Hence $\chi \psi$ must also have conductor $p^{\alpha}$ so $G(\chi \psi) \neq 0$, and thus $T(\chi) \neq 0$.

The proof of the proposition is now complete.

Our goal here is to give an analog of Theorem 2 in Section 3 for the minimal polynomial $G(X)$ for the reciprocals of the $\psi_{j}$ in (26). Two situations arise naturally depending on whether $(p-1) / f$ is even or odd. To deal with the case $(p-1) / f$ is even, we set

$$
\hat{\beta}_{f}(n)= \begin{cases}\beta_{f}(n) & \text { if } n \text { is even } \\ 0 & \text { if } n \text { is odd }\end{cases}
$$


where $\beta_{f}(n)$ is the counting function in (15). In terms of this adjusted counting function $\hat{\beta}_{f}(n)$ and the exceptional sets $\xi_{f}(n)$ as before, we find

Theorem 3. Suppose $(p-1) / f$ is even where $\hat{B}(X)=\exp \left(-\sum_{n=1}^{\infty} \hat{\beta}_{f}(n) X^{n} / n\right)$ with $\hat{\beta}_{f}(n)$ defined by $(27)$. Then for each natural number $N$, the congruence

$$
G(X) \equiv \hat{B}\left(i^{*} \sqrt{p} X\right)^{\phi\left(p^{\alpha}\right) / f} \quad\left(\bmod X^{N}\right)
$$

holds in $\mathbf{Z}[X]$ for all odd prime powers $p^{\alpha}(\alpha>1)$ except those lying in $\xi_{f}(n)$ for some $n<N$.

Proof. Here $\log G(X)=-\sum_{n=1}^{\infty} S_{n} X^{n} / n$ takes the form

$$
-\sum_{n=1}^{\infty} \frac{\left(i^{*} \sqrt{p} X\right)^{n}}{n}\left(\sum_{j=1}^{e}\left(\sum_{H}\left(\frac{t_{j} x}{p}\right) \zeta_{p^{\alpha}}^{t_{j} x}\right)^{n}\right)
$$

with inner sum

$$
\sum_{j=1}^{e}\left(\sum_{H}\left(\frac{t_{j} x}{p}\right) \zeta_{p^{\alpha}}^{t_{j} x}\right)^{n}=\frac{1}{f} \sum_{r \in Z_{p^{\alpha}}^{*}}\left(\sum_{x \in H}\left(\frac{r x}{p}\right) \zeta_{p^{\alpha}}^{r x}\right)^{n}=\frac{1}{f} \sum_{r \in Z_{p^{\alpha}}^{*}}\left(\frac{r}{p}\right)^{n}\left(\sum_{H} \zeta_{p^{\alpha}}^{r x}\right)^{n},
$$

since $H$ is contained in $Z_{p^{\alpha}}^{* 2}$ as $(p-1) / f$ is even. When $n$ is even, this last sum is just

$$
\frac{1}{f} \sum_{r \in Z_{p^{\alpha}}^{*}}\left(\sum_{H} \zeta_{p^{\alpha}}^{r v}\right)^{n}=\frac{1}{f}\left(p^{\alpha} T_{n}\left(p^{\alpha}\right)-p^{\alpha-1} T_{n}\left(p^{\alpha-1}\right)\right)=\frac{\phi\left(p^{\alpha}\right)}{f} \beta_{f}(n)
$$

as in the proof of Theorem 2 when $p^{\alpha}$ does not lie in $\xi_{f}(n)$ for $n<N$. When $n$ is odd this sum becomes

$$
\frac{1}{f} \sum_{r \in Z_{p^{\alpha}}^{*}} \sum_{x_{i} \in H}\left(\frac{r}{p}\right) \zeta_{p^{\alpha}}^{r\left(x_{1}+\cdots+x_{n}\right)}=\frac{1}{f} \sum_{x_{i} \in H} \sum_{r \in Z_{p^{\alpha}}^{*}}\left(\frac{r}{p}\right) \zeta_{p^{\alpha}}^{r\left(x_{1}+\cdots+x_{n}\right)}=0
$$

for any prime power $p^{\alpha}$ not in $\xi_{f}(n)$ for some $n<N$, since $\sum_{r \in Z_{p^{\alpha}}^{*}}\left(\frac{r}{p}\right) \zeta_{p^{\alpha}}^{r v}=0$ unless $p^{\alpha-1} \| v$. This establishes the theorem with $\hat{B}(X)$ defined in terms of the adjusted counting function $\hat{\beta}_{f}(n)$ in $(27)$.

Example 3. Consider $p=7$ with $f=3$, so

$$
\tilde{\beta}_{3}(n)= \begin{cases}n ! /((n / 3) !)^{3} & \text { if } 6 \mid n, \\ 0 & \text { otherwise, }\end{cases}
$$

in (27) with

$$
\tilde{B}(X)=1-15 X^{6}-2775 X^{12}-910202 X^{18}-\cdots
$$

in Theorem 3. Then

$$
\tilde{B}(i \sqrt{7} X)=1+7^{3} \cdot 15 X^{6}-7^{6} \cdot 2775 X^{12}+7^{9} \cdot 910202 X^{18}-\cdots .
$$

One may again take $H=\{1,18,-19\}$ for $m=49$ or 343 as in Example 1, where 49 first appears in $\xi_{3}(4)$ and 343 first appears in $\xi_{3}(11)$. The minimal polynomial for the reciprocal of $\psi_{1}=i * \sqrt{7}\left(\zeta_{49}+\zeta_{49}^{18}+\zeta_{49}^{-19}\right)$ begins

$$
1+\underline{7}^{3} X^{4}+\underline{7}^{4} \cdot \underline{2} X^{5}+\underline{7}^{4} \cdot \underline{29} X^{6}+\cdots
$$

whereas that for the reciprocal of $\psi_{1}=i * \sqrt{7}\left(\zeta_{343}+\zeta_{343}^{18}+\zeta_{343}^{-19}\right)$ begins

$$
1+7^{5} \cdot 36 X^{6}-\underline{7}^{8} \cdot \underline{165} X^{11}+7^{9} \cdot 2325 X^{12}+\cdots .
$$


The underscored coefficients deviate as expected from the pattern given in Theorem 3 .

Now consider the case $(p-1) / f$ is odd, so $f$ must be even. For any positive integer $n$, let $\beta_{f}^{+}(n)$ count the number of times a sum satisfies $\epsilon_{1}+\cdots+\epsilon_{n}=0$ for $f$-roots of unity $\epsilon_{i}$ in $\mathbf{Q}\left(\zeta_{f}\right)$ with an even number of the $\epsilon_{i}$ actually being $f / 2$-roots of unity. Similarly, let $\beta_{f}^{-}(n)$ count the number of times a sum satisfies $\epsilon_{1}+\cdots+\epsilon_{n}=0$ for $f$-roots of unity $\epsilon_{i}$ in $\mathbf{Q}\left(\zeta_{f}\right)$ with an odd number of the $\epsilon_{i}$ actually being $f / 2$-roots of unity. Clearly $\beta_{f}^{+}(n)+\beta_{f}^{-}(n)=\beta_{f}(n)$ for any $n$, and also $\beta_{f}^{+}(n)=\beta_{f}^{-}(n)$ if $n$ is odd. Define an adjusted counting function $\hat{\beta}_{f}(n)$ by

$$
\hat{\beta}_{f}(n)=\beta_{f}^{+}(n)-\beta_{f}^{-}(n)
$$

for any positive integer $n$. Note that $\hat{\beta}_{f}(n)=0$ if $n$ is odd.

In terms of this adjusted counting function and exceptional sets $\xi_{f}(n)$ as before, we find

Theorem 4. Suppose $(p-1) / f$ is odd where $\hat{B}(X)=\exp \left(-\sum_{n=1}^{\infty} \hat{\beta}_{f}(n) X^{n} / n\right)$ with $\hat{\beta}_{f}(n)$ defined by (28). Then for each natural number $N$, the congruence

$$
G(X) \equiv \hat{B}\left(i^{*} \sqrt{p} X\right)^{\phi\left(p^{\alpha}\right) / f} \quad\left(\bmod X^{N}\right)
$$

holds in $\mathbf{Z}[X]$ for all odd primes powers $p^{\alpha}(\alpha>1)$ except those lying in $\xi_{f}(n)$ for some $n<N$.

Proof. In this case the inner sum in the expansion for $\log G(X)$ in the proof of Theorem 3 can be expressed

$$
\begin{aligned}
& \left.\sum_{j=1}^{e}\left(\sum_{H}\left(\frac{t_{j} x}{p}\right) \zeta_{p^{\alpha}}^{t_{j} x}\right)^{n}\right)=\frac{1}{f} \sum_{r \in Z_{p^{\alpha}}^{*}}\left(\sum_{x \in H}\left(\frac{r x}{p}\right) \zeta_{p^{\alpha}}^{r x}\right)^{n} \\
& \quad=\frac{1}{f} \sum_{x_{i} \in H}^{+} \sum_{r \in Z_{p^{\alpha}}^{*}}\left(\frac{r}{p}\right)^{n} \zeta_{p^{\alpha}}^{r\left(x_{1}+\cdots+x_{n}\right)}-\frac{1}{f} \sum_{x_{i} \in H}^{-} \sum_{r \in Z_{p^{\alpha}}^{*}}\left(\frac{r}{p}\right)^{n} \zeta_{p^{\alpha}}^{r\left(x_{1}+\cdots+x_{n}\right)}
\end{aligned}
$$

since $\left(\frac{x}{p}\right)=-1$ for any $x \in H-H^{2}$. Here $H^{2}$ is just the set of $f / 2$-roots of unity in $\mathbf{Z}_{p^{\alpha}}^{*}, \sum^{+}$is the sum over tuples $\left(x_{1}, \ldots, x_{n}\right)$ with an even number of components lying in $H-H^{2}$ and $\sum^{-}$is the analogous sum over tuples $\left(x_{1}, \ldots, x_{n}\right)$ with an odd number of components lying in $H-H^{2}$. If $p^{\alpha}$ is not in any $\xi_{f}\left(n^{\prime}\right)$ for $n^{\prime}<N$, then each term $\sum_{r \in Z_{p^{\alpha}}^{*}}\left(\frac{r}{p}\right) \zeta_{p^{\alpha}}^{r\left(x_{1}+\cdots+x_{n}\right)}=0$ in (29) and hence $S_{n}=0$ when $n$ is odd, since no sum $x_{1}+\cdots+x_{n}$ is exactly divisible by $p^{\alpha-1}$. When $n$ is even, there may be sums $x_{1}+\cdots+x_{n}$ divisible by $p^{\alpha}$, each such sum $\sum_{r \in Z_{p^{\alpha}}^{*}} \zeta_{p^{\alpha}}^{r\left(x_{1}+\cdots+x_{n}\right)}$ contributing $\phi\left(p^{\alpha}\right)$ in (29). Overall, there are $\beta_{f}^{+}(n)$ of these with a + sign and $\beta_{f}^{-}(n)$ with a $-\operatorname{sign}$, so $(29)$ matches $\frac{\phi\left(p^{\alpha}\right)}{f}\left(\beta_{f}^{+}(n)-\beta_{f}^{-}(n)\right)$. This establishes the assertion of the theorem with $\hat{B}(X)$ defined in terms of the adjusted sums $\hat{\beta}_{f}(n)$ in (28). 
Example 4. Consider $p=7$ with $f=6$. Here one finds $\beta_{6}^{+}(1)=\beta_{6}^{-}(1)=0$, $\beta_{6}^{+}(2)=0, \beta_{6}^{-}(2)=6, \beta_{6}^{+}(3)=\beta_{6}^{-}(3)=6, \beta_{6}^{+}(4)=90, \beta_{6}^{-}(4)=0, \beta_{6}^{+}(5)=$ $\beta_{6}^{-}(5)=180, \beta_{6}^{+}(6)=180$ and $\beta_{6}^{-}(6)=-1860$ to obtain values $\hat{\beta}_{6}(2)=-6$, $\hat{\beta}_{6}(4)=90$ and $\hat{\beta}_{6}(6)=-1680$ in $(28)$. Then

$$
\hat{B}(X)=1+3 X^{2}-18 X^{4}+217 X^{6}+\cdots
$$

in Theorem 4 so

$$
\hat{B}(i \sqrt{7} X)=1-21 X^{2}-18 \cdot 7^{2} X^{4}-217 \cdot 7^{3} X^{6}-\cdots .
$$

One may take $H=\{ \pm 1, \pm 18, \pm 19\}$ for $m=49$ or 343 . From Table 2 one finds that 49 first appears in $\xi_{6}(3)$, whereas 343 first appears in $\xi_{6}(8)$. The minimal polynomial for the reciprocal of $\psi_{1}=i \sqrt{7}\left(\zeta_{49}+\zeta_{49}^{18}+\zeta_{49}^{-19}-\zeta_{49}^{-1}-\zeta_{49}^{-18}-\zeta_{49}^{19}\right)$ in (26) is

$$
1-7^{2} \cdot 3 X^{2}-\underline{7}^{3} X^{3}+\underline{7}^{4} X^{4}+\underline{2} \cdot \underline{7}^{4} X^{5}-\underline{7}^{4} X^{7},
$$

whereas that for the reciprocal of $\psi_{1}=i \sqrt{7}\left(\zeta_{343}+\zeta_{343}^{18}+\zeta_{343}^{-19}-\zeta_{343}^{-1}-\zeta_{343}^{-18}-\zeta_{343}^{19}\right)$ begins

$$
1-7^{3} \cdot 3 X^{2}-7^{4} \cdot 198 X^{4}-7^{6} \cdot 1111 X^{6}+\underline{7}^{7} \cdot \underline{29027} X^{8}+\underline{7}^{8} \cdot \underline{6} X^{9}+\cdots .
$$

The underscored coefficients deviate as expected from the pattern of the beginning coeficients given in Theorem 4.

Example 5. Next consider $p=5$ with $f=4$. One readily finds that

$$
\hat{\beta}_{4}(n)= \begin{cases}\left(\begin{array}{c}
n \\
n / 2
\end{array}\right)^{2} & \text { if } n \text { is even } \\
0 & \text { if } n \text { is odd }\end{cases}
$$

in (28) so

$$
\hat{B}(X)=1-2 X^{2}-7 X^{4}-50 X^{6}-456 X^{8}-\cdots
$$

in Theorem 4 and hence

$$
\hat{B}(\sqrt{5} X)=1-10 X^{2}-5^{2} \cdot 7 X^{4}-5^{5} \cdot 2 X^{6}-5^{4} \cdot 456 X^{8}-\cdots .
$$

Here we take $H=\{ \pm 1, \pm 7\}$ for $m=25$ and $H=\{ \pm 1, \pm 57\}$ for $m=125$ again as in Example 2, where 25 first appears in $\xi_{4}(3)$ and 125 first appears in $\xi_{4}(7)$. The minimal polynomial for the reciprocal of $\psi_{1}=\sqrt{5}\left(\zeta_{25}+\zeta_{25}^{-1}-\zeta_{25}^{7}-\zeta_{25}^{-7}\right)$ is

$$
1-50 X^{2}+\underline{125} X^{3}+\underline{125} X^{4}-\underline{500} X^{5},
$$

whereas that for the reciprocal of $\psi_{1}=\sqrt{5}\left(\zeta_{125}+\zeta_{125}^{-1}-\zeta_{125}^{57}-\zeta_{125}^{-57}\right)$ begins

$$
1-250 X^{2}+5^{4} \cdot 41 X^{4}-5^{7} \cdot 18 X^{7}-\underline{5}^{7} X^{7}+\cdots .
$$

The underscored coefficients deviate as expected from the pattern given in Theorem 4 .

One may have noticed that $\hat{\beta}_{4}(n)=\beta_{4}(n)$ in the last example. More generally,

Proposition 3. For $\nu>1, \hat{\beta}_{2^{\nu}}(n)$ in (28) satisfies $\hat{\beta}_{2^{\nu}}(n)=\beta_{2^{\nu}}(n)$. 
Proof. We first note that no sum of an odd number of $2^{\nu}$-roots of unity for $\nu \geq 1$ can vanish since each such root of unity is congruent to $1 \bmod \pi_{2}$, where $\pi_{2}$ is the unique prime above 2 in $\mathbf{Q}\left(\zeta_{2^{\nu}}\right)$. Now we assert no sum of an odd number of primitive $2^{\nu}$-roots of unity can lie in $\mathbf{Q}\left(\zeta_{2^{\nu-1}}\right)$ for $\nu>1$. Suppose to the contrary a sum $\zeta_{2^{\nu}}^{i_{1}}+\cdots+\zeta_{2^{\nu}}^{i_{n}}$ lies in $\mathbf{Q}\left(\zeta_{2^{\nu-1}}\right)$, where $\nu>1$ and the $i_{j}$ and $n$ are odd. Then $\zeta_{2^{\nu}}\left(\zeta_{2^{\nu-1}}^{\left(i_{1}-1\right) / 2}+\cdots+\zeta_{2^{\nu-1}}^{\left(i_{n}-1\right) / 2}\right)$ lies in $\mathbf{Q}\left(\zeta_{2^{\nu-1}}\right)$ so $\zeta_{2^{\nu-1}}^{\left(i_{1}-1\right) / 2}+\cdots+\zeta_{2^{\nu-1}}^{\left(i_{n}-1\right) / 2}=0$ contradicting our first remark. It follows easily now that for $\nu>1$ any sum of an even number of $2^{\nu}$-roots of unity which vanishes must always contain an even number of primitive $2^{\nu}$-roots of unity. Hence $\beta_{2^{\nu}}^{-}(n)=0$ for $n$ even. The statement of the proposition readily follows.

We conclude this section with a remark concerning the case $f=2$ which is not governed by the last proposition. One finds in this case that the counting function $\hat{\beta}_{2}(n)=\beta_{2}(n)$ in $(27)$ whenever $p \equiv 1(\bmod 4)$, whereas $\hat{\beta}_{2}(n)=(-1)^{[n / 2]} \beta_{2}(n)$ in $(28)$ for $p \equiv 3(\bmod 4)$. In each case, the respective power series $\hat{B}(X)=$ $\exp \left(-\sum_{n=1}^{\infty} \hat{\beta}_{2}(n) X^{n} / n\right)$ in Theorems 3 or 4 satisfies

$$
\hat{B}\left(i^{*} X\right)=\exp \left(-\sum_{n=1}^{\infty}\left(\begin{array}{c}
2 n \\
n
\end{array}\right) \frac{x^{2 n}}{2 n}\right)=\frac{1}{2}\left(1+\sqrt{1-4 X^{2}}\right) .
$$

In particular for the case $f=2$, one finds for any odd prime power $p^{\alpha}, \alpha>1$ that the congurence

$$
G(X) \equiv B(\sqrt{p} X)^{\phi\left(p^{\alpha}\right) / 2} \bmod X^{N}
$$

holds in $\mathbf{Z}[X]$ for $N=p^{\alpha-1}$ but not for $N>p^{\alpha-1}$, where

$$
B(X)=\exp \left(-\sum_{n=1}^{\infty}\left(\begin{array}{c}
2 n \\
n
\end{array}\right) \frac{X^{2 n}}{2 n}\right) .
$$

Indeed, we had previously remarked in Section 3 that $p^{\alpha}$ first appears in an exceptional set $\xi_{2}(n)$ for $n=p^{\alpha-1}$. One consequence is that the first $p^{\alpha-1}$ coefficients of $G(X)$ satisfy

$$
c_{r}= \begin{cases}(-1)^{r / 2} p^{r / 2} \frac{\phi\left(p^{\alpha}\right)}{\phi\left(p^{\alpha}\right)-r}\left(\begin{array}{c}
\phi\left(p^{\alpha}\right) / 2-r / 2 \\
r / 2
\end{array}\right) & \text { if } r \text { is even } \\
0 & \text { if } r \text { is odd }\end{cases}
$$

for $1 \leq r<p^{\alpha-1}$ (chiefly, Corollary 3 in [9]). While (31) is not enough to determine $G(X)$, we have recently found a closed form formula for the coefficients of $G(X)$ analogous to (25) expressed in terms of an Aurifeuillian factor [3] of the cyclotomic polynomial $\psi_{p}$. The reader is referred to [9] for details.

\section{REFERENCES}

[1] B.C. Berndt, R.J. Evans and K.S. Williams, Gauss and Jacobi sums, Wiley-Interscience, New York, (1998). MR1625181(99d:11092)

[2] Z. Borevich and I. Shafarevich. Number Theory, Academic Press, New York, (1966). MR0195803 (33:4001)

[3] R.P. Brent, "On computing factors of cyclotomic polynomials," Math. Comp. 61 (1993), 131-149. MR.1205459 (93m:11131)

[4] L.E. Dickson, Elementary Theory of Equations, Wiley, New York.

[5] C.F. Gauss, Disquisitiones Arithmeticae, Yale University Press, New Haven, (1966). MR0197380 (33:5545) 
ON THE MINIMAL POLYNOMIAL OF GAUSS PERIODS FOR PRIME POWERS

[6] S. Gupta and D. Zagier, "On the coefficients of the minmal polynomial of Gaussian periods," Math. Comp. 60 (1993), 385-398. MR.1155574 (93d:11086)

[7] S. Gurak, "Minimal polynomials for Gauss circulants and cyclotomic units," Pac. J. Math. 102 (1982), 347-353. MR0686555 (84c:10032)

[8] S. Gurak, "Minimal polynomials for circular numbers," Pac. J. Math. 112 (1984), 313-331. MR0743988 (85i:11107)

[9] S. Gurak, "Minimal polynomials for Gauss periods with f=2," Acta Arith. 121 (2006), 233257.

[10] S. Gurak, "Explicit evaluation of multi-dimensional Kloosterman sums for prime powers" (to appear).

[11] H. Hasse, Vorlesungen uber Zahlentheorie, Springer-Verlag, Berlin, (1950). MR0051844 $(14: 534 \mathrm{c})$

[12] J. Neukirch, Class Field Theory, Springer-Verlag, New York, (1986). MR0819231|(87i:11005)

[13] H. Salie, "Uber die Kloostermanschen Summen S(u,v:q)," Math. Z. 34 (1932), 91-109. MR 1545243

Department of Mathematics, University of San Diego, San Diego, California 92110

E-mail address: gurak@sandiego.edu 